\title{
The Golden Hour Trauma Care
}

\author{
Subbiah Venkatesh Babu ${ }^{1}$ \\ ${ }^{1}$ Department of Orthopaedic \& Trauma, Sri Sakthi Hospital, \\ Tirunelveli City, Tamil Nadu, India \\ Indian J Neurotrauma 2023;20:49-54.
}

\begin{abstract}
Address for correspondence Dr. Subbiah Venkatesh Babu, MB BS, MCh Orth., FRCS, Department of Orthopaedic \& Trauma, Sri Sakthi Hospital, 48 F, St Xavier's Colony, South Bypass Road, Tirunelveli 627 005, Tamil Nadu, India (e-mail: drsvbabu@hotmail.com).
\end{abstract}

\begin{abstract}
Keywords

- prehospital

- trauma

- injury

- golden hour

Globally the road accidents had become a great burden and claiming lot of precious lives today. However, the initial treatment within the first hour of the injury indeed had proven the high chance of survival after the trauma. This article updates and signifies the systematic emergency approach and current principles in saving lives after injury.
\end{abstract}

\section{Introduction}

Our world faces extensive amount of trauma-related morbidity and mortality every day. India adds more lives in the road accidents. The care of injured in all scenarios like road traffic, assault, or in disaster situation is getting viewed sincerely by the Indian Medical Association and by the Government today. ${ }^{1}$

Every doctor irrespective of the specialty needs to have the knowledge in life support and skills in saving the trauma victims. The honorable Supreme Court of India has directed all health establishments in our country to provide initial care for emergency and trauma victims and not to refuse treatment. $^{2}$ The Central and State Governments are taking significant initiatives in road safety and trauma care. The "Platinum ten minutes" (immediate 10 minutes from the minute of injury) is the purview of first responders including fire rescue officers, police, paramedics, and knowledgeable bystanders with initial stabilization and bringing the victims to the nearest clinical establishments irrespective of the dimension (scoop and run). Providing the golden hour trauma care (very first hour after injury) and shifting them to the higher center after stabilization is doctors' responsibility. However, the golden hour is individualized whether it is minutes or entire hour as per the injuries and needs of each patient. ${ }^{3,4}$

It is well proven that the initial timely care will increase the chance of survival of the trauma victims and of course significantly reduce the morbidity after injury. Patients with

received

May 8, 2020

accepted after revision

June 8,2020

article published online

February 22, 2021 life threatening injuries are increasing today. When these critically ill patients get the following initial life saving measures promptly before reaching the hospital, then the possibility of their survival is good.

\section{Prehospital Trauma Care}

\section{The steps involved in prehospital trauma care are as follows:}

- Clearing the airway.

- Stopping the bleeding.

- Supporting the fractured limbs.

- Shifting the victims safely to the nearest clinical establishments.

When the trauma patients arrive at the clinical establishments, every clinician while taking care of the trauma victims should comprehend following subjects well.

\section{Systemic Response to Trauma}

Any injured patient will have a local and a systemic response which is the real disease of trauma. Conventionally, this response is described as an initial catabolic, hypermetabolic phase, followed by an anabolic phase of recovery, all largely due to the effect of the endocrine response to trauma which is far more complex.

It consists of multiple linked responses or cascades with multiple alternative pathways so that intervention in one response usually leads to bypass others. We should intervene
DOI https://doi.org/ 10.1055/s-0040-1718479. ISSN 0973-0508. (c) 2021. The Author(s).

This is an open access article published by Thieme under the terms of the Creative Commons Attribution License, permitting unrestricted use, distribution, and reproduction so long as the original work is properly cited. (https://creativecommons.org/licenses/by/4.0/)

Thieme Medical and Scientific Publishers Pvt. Ltd., A-12, 2nd Floor, Sector 2, Noida-201301 UP, India 
to stop the process at source, i.e., stopping the hemorrhage, correcting the hypoxia, and clearing the contamination as soon as possible after injury and support organ function till the recovery takes place.

\section{Trimodal Death Distribution in Trauma}

- The first peak of death occurs at the time of injury which happens in seconds to minutes of injury. The reasons mainly are apnea after severe head injury, high spinal cord injury, rupture of the heart, and major vessels. Only prevention of trauma can reduce this peak of traumarelated deaths.

- The second peak lasts from the end of this first peak to several hours after the injury has taken place. Deaths that occur during this period are usually due to subdural and epidural hematoma, hemopneumothorax, splenic rupture, liver injury, pelvic fracture, and other injuries which contribute to major blood loss.

- The third peak of trauma death occurs several days or weeks after the initial injury. It is often due to sepsis and multiorgan failure.

The development of standardized trauma training, better prehospital care, and the implementation of trauma care systems with established protocols to care for injured patients will appreciably alter the said picture. The awareness of trauma care and all the facts related to trimodal distribution of mortality and their morbidity after injury, significance of medications, blood transfusion, longevity of hospital stay, and rehabilitation should be explained to the society well. This strategy has to be applied by the medical professionals in association with the government and nongovernment organizations.

\section{In-Hospital Golden Hour Trauma Care}

Every physician/doctor/specialist should be acquainted with the following important aspects of trauma care which are the "MIST"-from the ambulance paramedical staff, primary survey, resuscitate and re-evaluate, secondary survey, definitive care, tertiary survey, cardiopulmonary resuscitation (CPR), and safe transportation of trauma victims.

\section{The "MIST" Handover}

M Mechanism of injury

I Injuries observed

$\mathrm{S}$ Vital signs

$\mathrm{T}$ Therapy established

\section{At the Clinical Establishment}

Primary survey and initial trauma care:

- Ascertaining a patent airway with cervical spine control.

- Sufficient ventilation.

- Maintaining circulation which includes cardiac function and intravascular volume.

- Evaluating the overall neurological status.

\section{Airway and Spinal Immobilization}

It is well proven that the initial timely care will increase the chance of survival of the trauma victims and of course significantly reduce the morbidity after injury.

In severe trauma, resuscitation and assessment are performed simultaneously to detect and treat conditions that may be rapidly fatal. Diagnosing a threatened airway, clearing it, and if necessary, providing a definitive airway is the first priority, because an obstructed (threatened) airway can be fatal within 3 to 5 minutes. Common causes are head injury-induced coma (Glasgow Coma Scale $[\mathrm{GCS}]<9$ ), severe shock (systolic blood pressure $<70 \mathrm{~mm} \mathrm{Hg}$ ), unstable fracture maxilla, bilateral fracture mandible, inhalational burns, and less commonly, tracheal or laryngeal injury.

\section{Airway Assessment}

- A talking patient has a clear airway.

- Apnoeic patients are dying hence immediate orotracheal intubation is warranted.

- Maintain the immobilization of spine until cleared.

- Look: Air hunger, restlessness, coma (unresponsive to painful stimuli), penetrating injury to the throat. Cyanosis is a late sign.

- Listen: Stridor, gurgling in the throat, snoring.

- Feel: Facial fractures, surgical emphysema of the neck.

- Decide: Threatened airway or not. If not threatened, provide oxygen by face mask. If threatened, provide a definitive airway.

\section{Breathing}

Once the airway is defined as clear, or controlled (cuffed tube in the trachea), one can assess and treat the seven threats to life in the chest. Most are due to deficient breathing. These are:

1. Tracheal injury

2. Tension pneumothorax

3. Sucking chest wound

4. Massive hemothorax

5. Flail chest + pulmonary contusion

6. Cardiac tamponade

7. Contained rupture of the aorta

Management for the threats to life are performed by looking, feeling, listening, and immediately acting as mentioned below:

- Look: For external injury to the neck or chest, cyanosis, air hunger, sucking chest wounds, asymmetry of chest movement.

- Feel: Trachea centra, surgical emphysema, fractured ribs, and flail segment.

- Listen: Air entry left and right.

- Decide: Is there a threat to life?

- Act: Needle thoracentesis or not, three-way dressing or not, intercostals drain or not, intubate and ventilate or not, pericardiocentesis or not.

- X-ray: The only other threat to life is a contained rupture of the aorta, which will be picked up on X-ray chest. 


\section{Treatment}

- Tracheal injury: Definitive airway.

- Tension pneumothorax: Needle thoracentesis (14 Jelco) into second interspace midclavicular line, followed by intercostals drain.

- Massive hemothorax: Intercostals drainage-immediate drainage $>100 \mathrm{~mL}=$ immediate thoracotomy. Clamping the drain is of no benefit.

- Flail chest: Three or more ribs fractured in two or more places. Intubation and ventilation depending on blood gas analysis.

$\left(\mathrm{PO}_{2} / \mathrm{FIO}<200, \mathrm{PCO}_{2}>45 \mathrm{~mm} \mathrm{Hg}\right.$, respiratory rate $>30 /$ min, use of accessory muscles on maximal $\mathrm{FIO}_{2}(65 \% \mathrm{X}$ face mask).

- Cardiac tamponade: Pericardiocentesis, or immediate thoracotomy, depending on facilities.

\section{Circulation}

You have to integrate a lot of facts simultaneously. To make lifesaving decisions and act rapidly, you need to follow a system:

1. Identify the presence or absence of shock.

2. Define the class of shock.

3. Define the cause of shock.

4. Volume resuscitation.

5. Act to control the cause.

- Presence or absence of shock: A patient with tachycardia $>100 /$ min after trauma is in shock until proven otherwise.

- Look for anxiety, restlessness, aggression, depressed level of consciousness, external hemorrhage, distending abdomen, and obvious fractures.

- Listen to the heart sounds (muffled or not, tachycardia or not), systolic blood pressure (BP), and peripheral pulses.

- Feel for scalp lacerations, trachea central or not, chest wall as above, abdomen tender or not, pelvis stable or not, limb fractures or not.

\section{Class of shock:}

Class 1: $<750-\mathrm{mL}$ blood loss. Requires control of the cause and crystalloid infusion.

Class 2: 750 to 1,500-mL blood loss. Requires urgent control of the cause and crystalloid infusion.

Class 3: 1,500 to 2,000-mL blood loss. Requires urgent surgical intervention to control the cause.

Treatment: Crystalloid infusion (2,000 mL maximum), you may consider colloid (2,000 $\mathrm{mL}$ maximum), then blood transfusion. By this time, the patient should be on his way to theater.

Class 4: Blood loss $>2,000 \mathrm{~mL}$. Most serious injuries are possible and consider ER thoracotomy in penetrating trauma.

The diagnosis of shock is based on clinical signs. Drop in BP is a late sign, indicating greater than $40 \%$ mortality.

\section{Identification of the Cause of Shock}

For trauma victims, $90 \%$ of the shock is due to hemorrhage shock. This is either: external/compressible, or internal/ noncompressible.

Cardiogenic shock occurs after cardiac tamponade, tension pneumothorax, rarely blunt cardiac injury, and in elderly patients, myocardial infarction (often precipitated by hemorrhagic shock). Septic shock may occur within 6 hours after injury-beware of the patient subjected to delayed transfer.

Neurogenic shock occurs after transecting the autonomic spinal fibers-e.g., high paraplegia.

The cause of shock should be controlled.

\section{Hemorrhagic Shock}

Hemorrhagic shock is either compressible or noncompressible. The immediate step is to control compressible hemorrhage, e.g., bleeding lacerations, gunshots limbs, fractures, and degloving injuries.

Immediate action is required!

Lacerations: Compressive bandage $\rightarrow$ pack with swabs and suture under tension.

Fracture: Reduce, immobilize splint, and apply compressive bandage to bleeding compound fractures.

Tourniquet is applied if it is a matter of life before limb, i.e., all other methods of hemorrhage control have failed, or BP $<70 \mathrm{~mm} \mathrm{Hg}$.

Pelvis: Stable or not, step over symphysis or not. If pelvis is unstable, circular compression can be achieved by tightly tying a sheet around the pelvis at the level of trochanters. Commercial binders are also available at other centers. If a vertical shear injury is noted, the hemipelvis should be reduced with traction first before circular compression. Medical anti shock trousers (MAST) suits (pneumatic antishock garments) are rarely available for use these days.

The above are all temporary measures-get the patient to the operation theater as soon as possible!

\section{Noncompressible Hemorrhage}

- Dictum: Intracranial injury is never a cause of hypotension; scalp injury is a common cause of hypotension.

- Neck: For penetrating neck injuries, put the patient in the Trendelenburg position to prevent air embolism, control the airway with an ET tube, inflate a Foleys catheter in the cavity, and suture the laceration under tension.

- Chest: See breathing-call a surgeon.

- Abdomen: See abdominal injuries-call a surgeon.

- Volume resuscitation: Immediately act in establishing two wide bore (14-16 G) venous access in the cubital fossa-if not injured and same time drawing blood for investigations. With hypovolemic shock, vascular access and volume replacement are critical to get time till bleeding can be controlled. Access to central veins can be achieved by means of internal jugular or subclavian vein puncture, the former being safer. In patients with neck or arm injuries the intravenous line should be inserted on the opposite side to avoid extravasation of 
the infused fluid from a proximal venous injury. The infusion rate depends on the length and diameter of the catheter and not on the size of the vein.

- Fluid therapy: Ringers lactate 1,000 to $2,000-\mathrm{mL}$ bolus intravenous (IV) stat, followed by maximum of $2,000-\mathrm{mL}$ colloid (Voluven, Gelofusine). Thereafter delusional coagulopathy occurs. BP is maintained at no more than $90 \mathrm{~mm} \mathrm{Hg}$ for penetrating injuries to avoid vasodilatation, dislodgement of clot, and rebleeding. Head injured patients need all the cerebral perfusion they can get to avoid secondary brain injury-keep the BP as normal as possible.

- Blood: Low titer $0 \mathrm{Rh}$ negative: no need for typing or crossmatching. For life-threatening blood loss only. Use of $0 \mathrm{Rh}$

+ blood acceptable for males.

- Red-labeled: Typing but no cross-matching. Ready in approximately 10 to 15 minutes.

- Crossmatch and hold: Available within 10 minutes once cross-matched, but not charged until called for.

- Fully typed and cross-matched: Ready in approximately 30 minutes.

Ordering the blood is guided by the severity of trauma and the patient's response to resuscitation. If more than 2 units of blood are required for resuscitation, then order blood products at the ratio of 1:1:1 for PRC:FFP:PLT (packed red blood cells: fresh frozen plasma: platelets). If whole blood is available, it is preferable. The massive blood transfusion can be modified later with the evaluation of coagulation system. At that stage other blood products like cryoprecipitate may be added. Leucocyte depleted red blood cells are preferred.

\section{Disability}

The aim is to define the immediate threats to life due to brain injury, spinal cord injury; and to protect against secondary brain or spinal cord injury (due to hypo perfusion or hypoxia). Concentrate head-and spinal cord injury. Following a protocol avoids missing injuries.

\section{Assessment of the Level of Consciousness (GCS)}

- Identifying localizing signs.

- Protecting the brain and spinal cord against secondary injury (oxygen and perfusion).

- Deciding not to obtain a CT-scan.

Assessment of the level of consciousness-GCS is a more elegant way; however, AVPU scale is the quickest way to assess the suspected neurological injury. ${ }^{5}$

\section{- AVPU}

\section{A: Alert.}

V: Responds to verbal stimuli only.

$P$ : Responds to painful stimuli only.

U: Unconscious and unresponsive to painful stimuli.

- Localizing signs.

- Pupils: Size and reaction.

- Limbs: Does the patient move all limbs or not.
- Protecting the brain.

- Intubate and ventilate if not responsive to painful stimuli (= GCS 8/15).

- Ventilate to PO2 >100, and PCO2 30 to 35.

- Maintain the systolic BP as high as possible.

- Consider mannitol IV.

- CT scan or not.

Mandatory for GCS $<14$, lateralizing signs, and epileptic fit.

It is advised for all significant head injury with loss of consciousness (depending on facilities). Especially in highrisk patients (on warfarin, antiplatelet therapy, bleeding disorders, elderly, worsening posttraumatic headaches, retrograde amnesia, etc.).

\section{Exposure}

By protocol, all clothing must be removed immediately to allow full examination during primary and secondary survey. If necessary, clothing is cut away.

Hypothermia is a fatal complication of trauma. Core temperature $<35^{\circ} \mathrm{C}$ increases the mortality of major trauma to $35 \%$ (from $18 \%$ ), and to $100 \%$ if core temperature is below $32^{\circ} \mathrm{C}$. Use warm fluid, warm gas, and a warm environment from the start. The external re-warming device of choice is a Bair Hugger. If not available, wrap the patient in plastic bags. If intubated, wrap the head as well. The head accounts for up to $28 \%$ of heat loss.

- Remove all clothing/exposure.

- At this stage, oxygen saturation monitor is applied.

- Two-wide bore lines, Ringers lactate IV at maximum speed.

- Arterial blood gas collected.

- Echocardiography monitor connected.

- Logroll, rectal, and perineal examination.

While logrolling, a rapid secondary survey of the back can be performed-see below:

Urinary catheter if perineum intact.

Nasogastric (or orogastric) tube if required.

Analgesia and sedation.

X-ray chest, pelvis, and focused assessment of sonogram in trauma (FAST scan).

\section{Secondary Survey and Continuing Resuscitation with Monitoring}

The patient is examined in detail from head to foot and front to back. If the patient stabilizes, then he/she has to be shifted safely to a higher center for further management with continuous monitoring and life support. If your clinical establishments have infrastructure to manage further, then a secondary survey and diagnostic studies are performed. However, if the patient remains unstable, he or she should be taken immediately to the operating room to achieve surgical hemostasis or to the surgical intensive care unit.

\section{Tertiary Survey and Documentation}

Full examination again with investigations are done so that injuries are not missed. The complete repetition of survey is important along with documentation. 


\section{Management of Penetrating and Crushed Trauma}

It is good practice to place metallic objects, such as paper clips, on the skin pointing to the various wounds on the chest wall, which aid the surgeon in determining the missile track. This also can be useful for stab wounds. Tracking the missile helps the surgeon to determine which visceral organs may be injured and, in particular, whether or not there is a potential transgression of the diaphragm and/or mediastinum. It is recommended that an "unfolded" paper clip could be placed on any anterior penetrating injury, and a "folded" one on any posterior injury.

\section{Amputations-Massive Limb Trauma: Life versus Limb}

Presently, the decision of amputation based on Ganga Hospital Open Injury Scoring system is made. ${ }^{6}$ The management of the mangled limb remains a multidisciplinary approach and involves the combined skills of the orthopaedic, vascular, plastic, and reconstructive surgeons. This will prevent persistent painful debility or an insensate or flail limb is still the outcome. Successful limb salvage is defined by the overall function and satisfaction of the patient.

\section{Management of Poly/Major Trauma ${ }^{7,8}$}

The ideology of the management for patients suffering from major trauma must be a multidisciplinary approach with following key essentials:

- Synchronized and repeated assessment with resuscitation.

- Diagnostic studies if the patient becomes hemodynamically stable.

- Lifesaving surgery/damage control surgery.

\section{Cardiopulmonary Resuscitation}

Whenever faced with a victim who seems to be dead/dying, CPR is an essential first step.

- Call for help if any is available.

- Tilt the head back and listen for breathing. If not breathing normally, pinch nose and cover the mouth with yours and blow until the chest rises. Give two breaths. Each breath should take 2 seconds.

- If the victim is still not breathing normally, coughing, or moving, begin chest compressions. Push down the chest between 1 and 0.5 to 2 inches 15 times right between the nipples. Pump at the rate of $100 / \mathrm{min}$.

- Continue with two breaths and 15 pumps until help arrives. This ratio is the same for one-person and twoperson CPR. In two-person CPR, the person pumping the chest stops while the other gives mouth-to-mouth breathing.

- Vomiting is the most frequently encountered complication of CPR. If the victim starts vomiting, turn the head to the side and try to sweep out or wipe off the vomit. Continue with CPR.

\section{In children}

- If alone with the child, give one minute of CPR before calling for help.

- Use the heel of one hand for chest compressions.

- Press the sternum down 1 to 1.5 inches.

- Give one full breath followed by five chest compressions.

\section{Prehospital Care with Triage and Safe Transporting Victims}

Under massive trauma incidents, priorities should be set judiciously amongst the injured victims preferring greater attention to more deserving individuals than the less deserving ones. This is "triaging"-sorting of patients based on the need for treatment and the available resources. Patients with the greatest chance of survival with the least expenditure of time, equipment, and personnel are to be managed first.

\section{Initial Care of Injured in Summary}

The golden hour trauma care is important, and the goal of initial trauma management is to restore adequate oxygenation and tissue perfusion in the shortest possible time, rapidly treating all the injuries well. The trauma mortality is not only associated with prehospital time but with injuries, transfers from primary care, probably deficient care. ${ }^{9}$ The trauma care is lifesaving but expensive. A structured trauma care system will reduce the cost and save many lives. ${ }^{10,11}$

To improve survival after major trauma, enhancement of resources for resuscitation and capacity building of doctors at all levels including primary centers to secondary and tertiary care centers should be the priority in all countries having more trauma. It has been much stressed by the World Health Organization with guidelines. ${ }^{11,12}$

Funding

None.

Conflict of Interest

None declared.

\section{References}

1 Road Safety PolicyGovernment of Tamil Nadu; 2007

$2 \mathrm{lgl}$. Parmanand Katara vs. Union of India. The Direction of Honourable Supreme Court of India; 1989/lgl

3 Rogers FB, Rittenhouse KJ, Gross BW. The golden hour in trauma: dogma or medical folklore? Injury 2015;46(04):525-527

4 Trauma Mortality and the Golden hour, CCC I Trauma I LITFL I; 2019. Available at: https://litfl.com/trauma-mortality-and-thegolden-hour/.

5 Romaneli D, Farrell MW. AVPU (Alert, Voice, Pain, Unresponsive). Treasure Island, FL: StatPearls Publishing; 2020

6 Rajasekaran S, Sabapathy SR, Dheenadhayalan Jet al.Ganga hospital open injury score in management of open injuries. Eur J Trauma Emerg Surg 2015;41(01):3-15

7 Major Trauma-NICE Guidelines, UK. Available at: https:// pathways.nice.org.uk/pathways/trauma/major-trauma 
8 Valentino TP. Major trauma: what is important for the best outcome and survival? J Postgrad Med 2017;63(03):149150

9 Dharap SB, Kamath S, Kumar V. Does prehospital time affect survival of major trauma patients where there is no prehospital care? J Postgrad Med 2017;63(03):169-175

10 Bonnie RJ, Fulco CE, Liverman CTInstitute of Medicine (U.S.) Committee on Injury Prevention and Control; Reducing the
Burden of Injury: Advancing Prevention and Treatment. Washington, DC: National Academies Press (U.S.)1999

11 Prehospital Trauma Care Systems, World Health Organisation. Available at: https://apps.who.int/iris/bitstream/handle/10665/ 43167/924159294X.pdf?sequence=1

12 Guidelines for Essential Trauma Care, World Health Organisation. Available at: https://www.who.int/violence_injury_prevention/ publications/services/guidelines_traumacare/en/ 